\title{
Thermal conductivity of free-standing silicon nanowire using Raman spectroscopy
}

\author{
Sandhyarani Sahoo, ${ }^{1,2}$ Sameer Kumar Mallik, ${ }^{1,2}$ Mousam Charan Sahu, ${ }^{1,2}$ Anjana Joseph, ${ }^{3}$ \\ Bibhudutta Rout, ${ }^{4}$ Gopal K. Pradhan, ${ }^{5 *}$ Satyaprakash Sahoo ${ }^{1,2 *}$ \\ ${ }^{1}$ Laboratory for Low Dimensional Materials, Institute of Physics, Bhubaneswar, 751005, India \\ ${ }^{2}$ Homi Bhabha National Institute, Training School Complex, Anushakti Nagar, Mumbai 400085, India \\ ${ }^{3}$ Jawaharlal Nehru Centre for Advanced Scientific Research, Jakkur, Bangalore, 560064, India \\ ${ }^{4}$ Department of Physics, University of North Texas, Denton, TX 76203, USA \\ ${ }^{5}$ Department of Physics, School of Applied Sciences, KIIT Deemed to be University, Bhubaneswar, \\ Odisha, 751024, India
}

\begin{abstract}
:
Low dimensional systems, nanowires, in particular, have exhibited excellent optical and electronic properties. Understanding the thermal properties in semiconductor nanowires is very important for their applications in their electronic devices. In the present study, the thermal conductivity of a freestanding silicon nanowire (NW) is estimated employing the Raman spectroscopy. The advantage of this technique is that the light source (laser) can be used both as heating and excitation source. The variations of the first-order Raman peak position of the freestanding silicon NW with respect to temperature and laser power are carried out. A critical analysis of effective laser power absorbed by exposed silicon NW, the detailed Raman study along with the concept of longitudinal heat distribution in silicon NW, the thermal conductivity of the freestanding silicon NW of 112 $\mathrm{nm}$ diameter is estimated to be $\sim 53 \mathrm{~W} / \mathrm{m} . \mathrm{K}$.
\end{abstract}

* Corresponding author: sahoo@iopb.res.in, gopal.pradhanfpy@kiit.ac.in 


\section{INTRODUCTION:}

Silicon-based semiconductor technology has brought a technological revolution towards the progress of our society, which has benefited tremendously from its versatile use and remarkable capabilities. The development of electronics based on silicon and its supporting technology has brought down the physical size of devices and interconnects to the nanoscale regime. In this connection, NWs in general and silicon NWs, in particular, are promising materials for the miniaturization of devices towards the nanoscale regime. ${ }^{1,2}$ Nowadays, the growth of NWs by different methods has been achieved with great control over their crystal quality, aspect ratio and heterostructures that has opened a new window to engineer various functional properties. ${ }^{3-7}$ In a world of ever-shrinking circuits size due to the integration of various miniaturized components such as NWs, the management, and transport of heat is paramount. It is quite important for better understanding and designing heat transporting devices in a host of applications, from heat management in high-power electronics, batteries, and photovoltaics to thermoelectric energy harvesting and solid-state cooling. For example, applications such as thermoelectric energy harvesting, solid-state cooling, and thermal barrier coatings would require extremely low thermal conductivity. On the contrary, heat management in high-power electronics, batteries, and photovoltaics would require high thermal conductivity. Understanding and predicting the thermal conductivity of nanosystems will allow designing heat transport for a wide range of technological applications. Determination of the thermal conductivity of silicon NWs plays a pivotal role in two of their most fundamental potential applications: (i) heat dissipation for potential heat drain application, and (ii) thermoelectric materials. In these applications a combination of lower thermal conductivity and high electrical conductivity can result in NWs having superior performance than existing materials presently used for thermoelectric refrigeration. ${ }^{8,9,10}$ A better understanding of thermal transport in nanostructures also has direct consequences in the design and performance of modern nanoelectronics for NW-based thermoelectric devices. Moreover, in the semiconducting NW system, the phonon contribution to the thermal conduction can be minimized by limiting the NW length scales smaller than phonon mean free path while keeping the contribution to the electrical conductivity unaltered. ${ }^{11}$ Thus, it is quite clear that prior determination of the thermal conductivity of the NWs is of extreme importance. 
Thermal conductivity in solids is due to the heat transfer by the electrons and phonons. ${ }^{12}$ The predominant carriers of heat in the case of semiconductors and insulators are the phonons. Reduction of the dimension of the bulk material into nanostructure results in the spatial confinement of the thermal carriers. Movement of these carriers in a confined geometry will result in increased phonon boundary scattering and phonon-phonon scattering rate. Considering these facts, thermal conductivity as a function of silicon NW diameter has been investigated using molecular dynamics simulations by Ponomareva et al. ${ }^{13}$ Their finding shows an interesting trend in thermal conductivity; predominant boundary scattering leads to suppressed thermal conductivity for NWs having diameter $>1.5 \mathrm{~nm}$ and phonon confinement effect results in enhanced thermal conductivity in silicon NWs having a diameter $<1.5 \mathrm{~nm} .{ }^{13}$

Measurement of the thermal conductivity of nanostructures such as graphene, carbon nanotubes, NWs, TMDCs is very challenging. In literature thermal conductivity of nanostructures is reported using micro heaters, ${ }^{14} 3 \omega$ method, ${ }^{15-17}$ Raman spectroscopy, ${ }^{18-23}$ time-domain thermoreflectance (TDTR). ${ }^{24}$ Among these techniques, Raman spectroscopy is found to be a simple and convenient technique to measure thermal conductivity has recently been applied to 2-D ${ }^{18-21}$ as well as 1-D nanostructures. ${ }^{22,23,25}$ The growing use of Raman thermometry arises from advantages lacking in other established techniques such as $3 \omega,{ }^{15,16}$ micro heating ${ }^{14}$ that are relatively complex, require direct contact with sample with the necessity of microfabrication. Unlike the other standard optical techniques that measures the thermal conductivity only in the cross-plane direction, Raman technique measures the in-plane thermal conductivity. As it has been found in literature that for nanostructures having anisotropy in particular directions, the measurement of in-plane thermal conductivity is more relevant. ${ }^{26}$ In Raman scattering method, the laser can be used both as heating and excitation source. ${ }^{27-29}$ Analyzing the shift of the Raman peak position caused by uniform rise in temperature and laser power induced local heating, the thermal conductivity can be estimated.

Recently, measurements of the thermal conductivity of individual silicon NW is reported by Joule heating ${ }^{14}$ or Raman thermometry method ${ }^{23}$. The Joule heating method is plagued by interfacial contact resistance as a potentially significant source of error, particularly with larger diameter NWs. In the second case, the NW was cantilevered. ${ }^{23}$ In this work, we present a relatively simple method to measure the thermal conductivity of freestanding single silicon NW by using Raman spectroscopy. The first-order temperature coefficient $(\delta \omega / \delta \mathrm{T})$ is obtained from the temperature- 
dependent Raman study and variation of Raman peak with laser power $(\delta \omega / \delta \mathrm{P})$ is determined by power-dependent Raman study and further analysis is carried out to calculate thermal conductivity.

\section{EXPERIMENTAL:}

Silicon wafer containing silicon NWs is commercially procured from CVD Materials Corporation, USA has grown by VLS CVD using First NanoTM Easytube ${ }^{\circledR}$ system. Microstructure characterization of silicon NWs is performed using Field Emission Scanning Electron Microscope (Zeiss make). Raman spectra silicon NWs are recorded using a confocal micro-Raman spectrometer (LABRAM HR Evolution, Horiba -Jobin-Yvon, France) with the $532 \mathrm{~nm}$ excitation of frequency-doubled Nd:YAG laser in the backscattering mode with an 1800 grooves/mm grating. The temperature-dependent Raman studies are carried out using a temperature stage (LINKAM THMS 600, Linkam Scientific Instruments, UK) with the help of a long working distance 50X objective. The power-dependent spectra in ambient temperature conditions are collected with a 100X objective $(\mathrm{NA}=0.9)$ by engaging discrete neutral density (ND) filters. For each ND filter, the final power was measured at the microscope objective end with a power meter (Thorlabs, USA).

Dense silicon NWs growth with a diameter varying between $100-500 \mathrm{~nm}$ with a large length (of several microns) can be easily noticed from figure 1 (a). Raman spectra are recorded on a bunch of NWs and a strong first-order Raman peak of silicon is observed as shown in figure 1 (b). It may be noted that the width of the Raman spectra is wider than bulk silicon. This NWs from the substrate are then transferred to a carbon-coated copper TEM grid. Once transferred, the grid is inspected for isolated single freestanding NW. This isolated individual NW can sometimes found to be suspended on ruptured carbon film where one end rests on the carbon film and another end lies on the copper grid. To minimize the role of contact on thermal conductivity we consider a suspended single NW of length much larger than the diameter of the laser spot. Figure 2 (a) shows an SEM image of such a freestanding silicon NW of diameter $\sim 112 \mathrm{~nm}$ with a suspended length of $\sim 7 \mu \mathrm{m}$. A careful analysis of the freestanding isolated NW indicates that the diameter of the NW is not uniform throughout its length i.e. from one side (right side) its diameter gradually decreases. Such tapering of diameter is expected for CVD grown large length NWs. However, for the present study for thermal conductivity measurement, the diameter can be approximated to be uniform. The suspended NW, laser heating are schematically shown on the left side of figure-2(b). 
We also schematically present the effective cross-sectional area of NW exposed to laser on the right side of figure-2(b). It can be clearly seen that the diameter of the laser spot is much larger as compared to the diameter of the suspended NW (details of which will be explained in a later section). The Raman spectra are collected from $77 \mathrm{~K}$ to $298 \mathrm{~K}$. The laser power is kept low in order to avoid local heating. The acquired spectra are fitted with Lorentzian lineshape to obtain the Raman peak positions and peak width (FWHM).
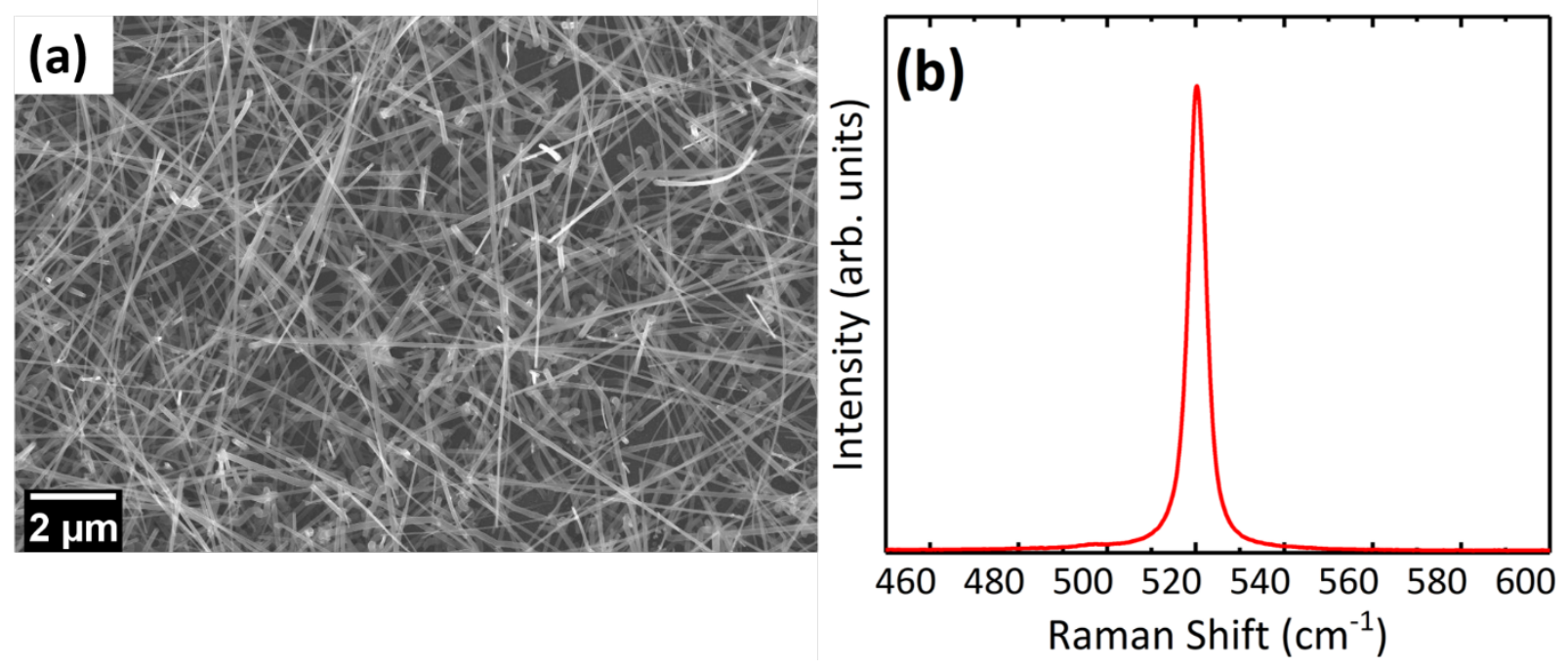

Figure-1: (a) SEM image of silicon NWs (b) Corresponding room temperature Raman spectra. 

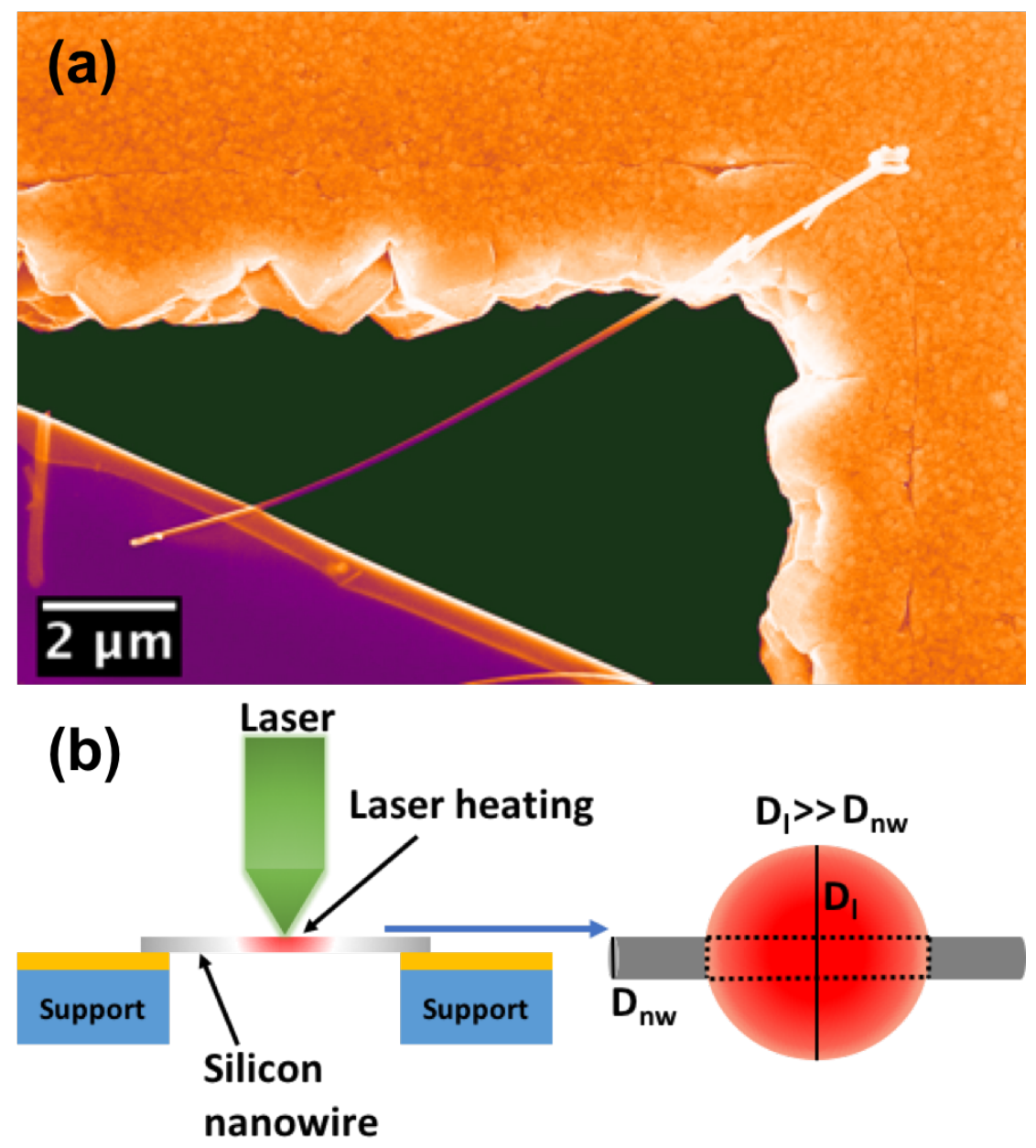

Figure-2:(a) False-colored SEM image of a freestanding single silicon NW on TEM grid (b) Schematic diagram of free-standing single silicon NW (left) and comparison of the dimension of silicon NW, laser spot size (right). The dotted rectangular section shows an effective crosssectional area of silicon NW exposed to the laser.

\section{RESULTS AND DISCUSSION:}

Thermal conductivity measurement using Raman spectroscopy would result in an inaccurate estimation for a sample directly placed on a substrate. This is due to the fact that as a part of the heat will dissipate to the substrate. In view of this most of the measurements have been carried out on a free-standing sample. ${ }^{18,19,22}$ As reported thermal conductivity estimation by Raman scattering requires a temperature and laser power-dependent studies. First, we carried out a temperaturedependent Raman study. The temperature of the sample is varied from 77 to $298 \mathrm{~K}$. It is worth arguing that high-temperature studies are deliberately eliminated in the present case because at 
high-temperature silicon samples could undergo oxidation resulting in a core-shell structure of $\mathrm{Si} / \mathrm{SiO}_{2}$. This can affect the temperature-dependent studies. Figure 3(a) shows the temperaturedependent Raman spectra. A systematic redshift of the first-order Raman peak position is noticed for the freestanding silicon NW with increasing temperature. This redshift is due to both the volume and temperature contribution as a consequence of anharmonicity of lattice vibration. The Raman peaks are well fitted with the Lorentzian lineshape and the extracted Raman peak position with the temperature are plotted in figure 3(b). The peak position as a function of temperature shows a linear relationship as fitted using $\omega=\omega_{0}+\alpha . T$, where $\omega_{0}$ is the frequency at $\mathrm{T}=300 \mathrm{~K}$, $\alpha$ is the first-order temperature coefficient. ${ }^{18,22}$ The slope of the curve gives the value of the firstorder temperature coefficient $(\alpha)$ and is found to be $-0.01776 \mathrm{~cm}^{-1} \mathrm{~K}^{-1}$. Doerk et al. ${ }^{30}$ have studied the variation of first-order temperature coefficient as a function of the diameter of silicon NW ranging from 20 to $200 \mathrm{~nm}$. The diameter dependent first-order temperature coefficient does not change significantly and our observed first-order temperature coefficient is also consistent with their reported result ${ }^{30}$

The broadening of the linewidth which is the measurer of phonon lifetime can be explained using the decay of phonon into two phonons or three phonons processes satisfying the conservation law. ${ }^{31,32}$ Considering a three phonons process, the line width can be expressed as,

$$
\Gamma(T)=\Gamma_{0}+E\left[1+\frac{2}{e^{x}-1}\right]+F\left[1+\frac{3}{e^{y}-1}+\frac{3}{\left(e^{y}-1\right)^{2}}\right]
$$

where $x=\frac{\hbar \omega}{2 . k_{B} \cdot T}, y=\frac{\hbar \omega}{3 . k_{B} \cdot T}, \Gamma_{0}$ is FWHM of the silicon NW in low temperature and $E, F$ is cubic and quartic anharmonic constants respectively. ${ }^{33}$ The variation of FWHM as a function of temperature is well fitted by the above equation for three phonon decay (Fig. 3 (b)) with cubic and quartic anharmonic constants of -0.3097 and 0.4375 respectively. 

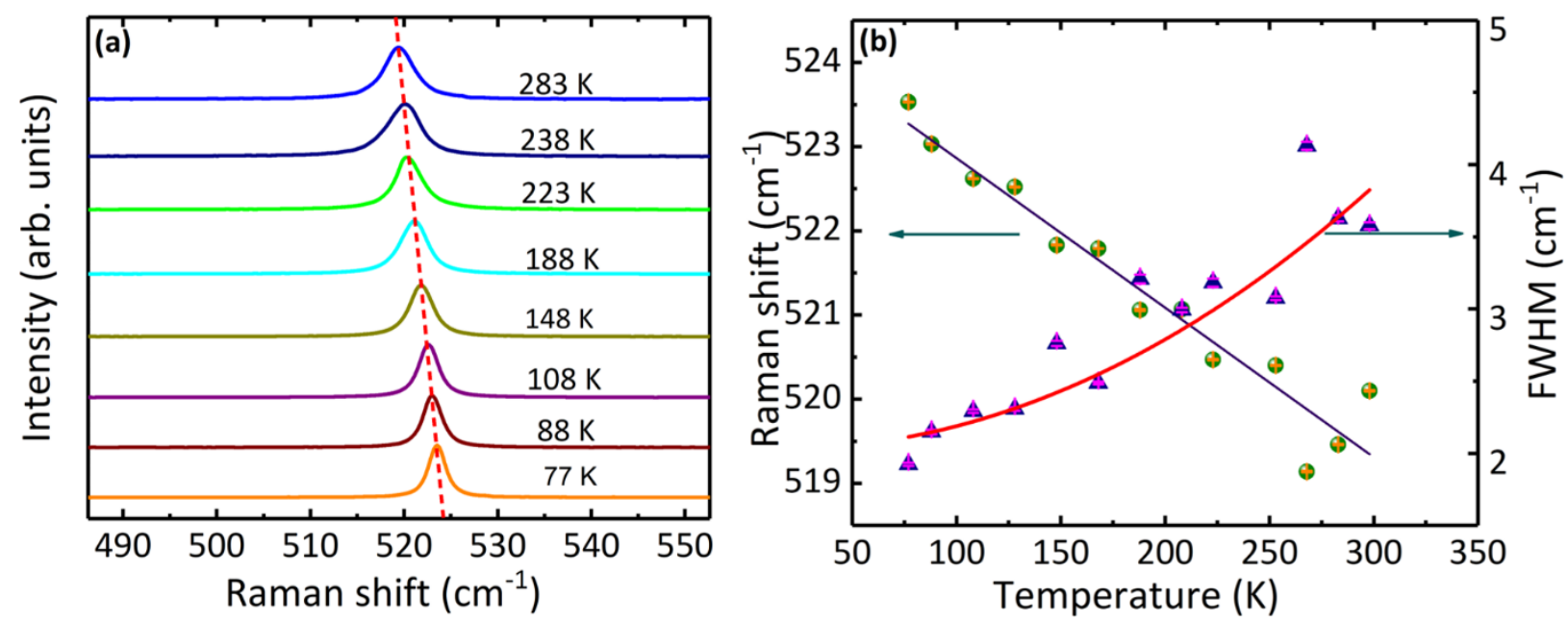

Figure-3: (a) First-order Raman spectra of freestanding silicon NW as a function of temperature. The dotted line is the guide to the eye. (b) Variation of Raman peak position and FWHM with temperature. Filled dots and filled triangles represent the peak position and FWHM, respectively. Solid lines are the fits to the experimental data.

Laser power dependent Raman studies with the variation of power is analogous to the temperaturedependent Raman spectra, it signifies that a strong laser power on the sample results in the localized heating. ${ }^{19}$ The thermal measurement requires a careful determination of the diameter of the laser beam spot $\left(D_{l}\right)$ and actual power absorbed by the NW. A theoretical estimation of the diffraction-limited spot size $\left(D_{l}=2 \lambda / \pi \mathrm{NA}\right)$ puts the lower limit on the spot size to be $0.38 \mu \mathrm{m}$ where $\lambda$ is the wavelength of the incident light $(532 \mathrm{~nm}), \mathrm{NA}$ is the numerical aperture of the objective lens (0.9), respectively. In our case, we assume the spot size to be $\sim 0.5 \mu \mathrm{m}$ which is much larger than the NW diameter $(112 \mathrm{~nm})$. As the laser beam spot is much larger than the silicon $\mathrm{NW}$, the power absorbed by the silicon NW is not the same as the incident laser power. During the experiment, the NW was placed carefully under the laser spot such that the diameter of the laser $\left(D_{l}\right)$ spot coincides with the NW, as schematically shown in figure 2 (b). Under such geometry, the cross-sectional area of the silicon NW illuminated by the laser is $D_{l} . D_{n w}$, where $D_{n w}$ is the diameter of the silicon NW. As mentioned our laser spot size is much larger than the diameter of silicon NW, we followed the effective power calculation of Doerk et al. ${ }^{23}$ The power absorbed 
$\left(P_{a}\right)$ by the silicon NW is $\frac{4 \cdot D_{n w} \cdot D_{l}}{\pi \cdot D_{l}^{2}}$ times the laser power $\left(P_{l}\right)$ and the absoptivity $(A)$ and can be expressed as follows.

$$
P_{a}=\frac{4 \cdot D_{n w} \cdot D_{l}}{\pi \cdot D_{l}^{2}} A \cdot P_{l}
$$

It may be noted here that the actual laser power as measured under the objective lens is varied from $43 \mu \mathrm{W}$ to $1.2 \mathrm{~mW}$ during the experiment. The absorptivity $(A)$ can be deduced using $A=$ $1-\frac{(n-1)^{2}+k^{2}}{(n+1)^{2}+k^{2}}$, where $n$ is the refractive index and $k$ is the extinction coefficient. ${ }^{34}$ For the laser wavelength of $532 \mathrm{~nm}$, the values of $n$ and $k$ are about 4.15 and 0.052 for bulk silicon ${ }^{35}$ giving the absorptivity $(A)$ of the silicon NW to $\sim 62 \%$. Using the above formula, the effective power $P_{a}$ is calculated to be in the range of $7 \mu \mathrm{W}$ to $201 \mu \mathrm{W}$. Figure 4 (a) shows the Raman spectra of the freestanding NW as a function of effective laser power.
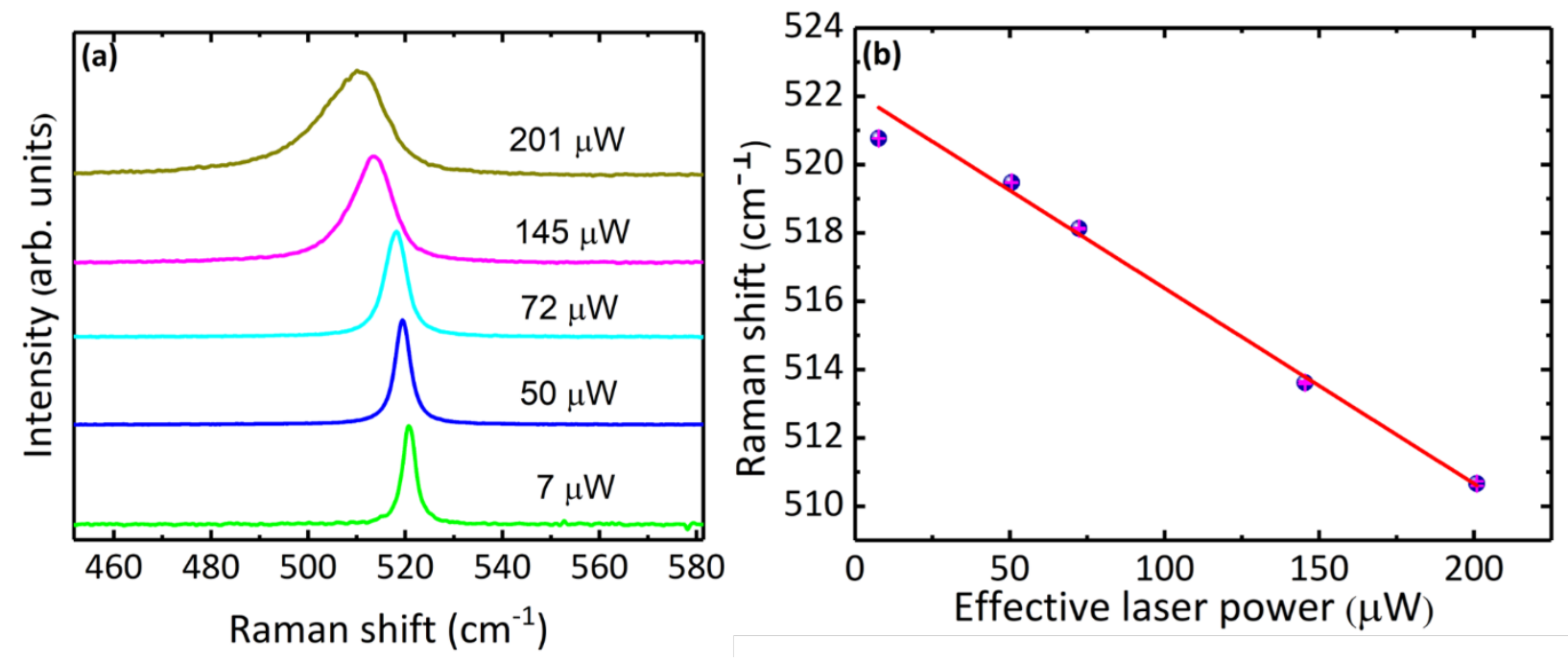

Figure-4: (a) Raman Spectra of the free-standing NW for different absorbed laser powers (b) Variation of Raman peak position with effective laser power. The solid line is a linear fit.

The symmetrical broadening of the Raman line and shifting of the first-order Raman peak position towards the lower wave-number side is also observed. The variation of Raman peak position with 
different power is shown in the Figure-4(b) and is fitted by a straight line and the slope $\left(\frac{\delta \omega}{\delta P_{a}}\right)$ of $\omega \sim P_{a}$ is found to be $-0.05721 \mathrm{~cm}^{-1}(\mu \mathrm{W})^{-1}$ from the linear fit.

Here, we would like to emphasize that silicon is always associated with a native oxide layer irrespective of its synthesis methods. The thickness of the oxide layer can vary depending on process conditions. The role of the oxide layer is very important in determining various properties of silicon NW. For an instant, during the electrical transport measurement of isolated silicon NW, the native oxide layer is removed carefully before making the electrical contacts. Being an insulator and highly dielectric material, the presence of silicon dioxide $\left(\mathrm{SiO}_{2}\right)$ layer on silicon $\mathrm{NW}$ can significantly affect its thermal properties. It has been experimentally observed that one requires higher laser power to get a significant Raman shift in silicon NW having a thicker oxide layer analogous to the thin oxide layer covered silicon NW. The work by Piscanec et al. ${ }^{36}$, Zixue Su et $a l .{ }^{33}$ shows shifts in the Raman spectra for both thin $(\sim 2 \mathrm{~nm})$ and thick $(\sim 10 \mathrm{~nm})$ oxide layers covering silicon NW respectively. In our case, significant changes in power-dependent Raman spectra are indicative of the existence of a very thin $(\sim 2-3 \mathrm{~nm})$ oxide layer covering the silicon NW.

Thermal conductivity of a sample can be determined by considering the heat distribution i.e. (i) radially heat distribution from the middle position of the sample towards the heatsink if the diameter of the sample is larger than the diameter of the laser spot (ii) longitudinal heat distribution from the middle position of the sample towards the heatsink if the diameter of the sample is comparable to the diameter of the laser spot. ${ }^{18}$ Transport of heat through the surface of a sample with a cross-sectional area $S$ is formulated as,

$$
\frac{\partial Q}{\partial t}=-\kappa \cdot \oint \nabla T \cdot d S
$$

where $\kappa$ is the thermal conductivity of the sample, $\frac{\partial Q}{\partial t}$ is the rate of heat transferred through the sample, $\nabla T$ is the temperature gradient. When the diameter of the sample is comparable to the diameter of the laser spot, the thermal conductivity of the sample can be formulated from the equation (3) as $\kappa=\frac{L}{2 . S} \cdot \frac{\Delta P_{l}}{\Delta T}$, where $\mathrm{L}$ is the distance from the laser-heated position of the sample to the heat sink and $\Delta T$ is the change in temperature due to the variation of the laser power. ${ }^{18}$ The 
value of $\mathrm{L}$ is about $\sim 3.4 \mu \mathrm{m}$ as measured from the SEM image [figure 2(a)]. We note that in our case the diameter of the laser spot is quite larger than the diameter of the silicon NW. However, if we consider the portion of the laser power $\left(\mathrm{P}_{\mathrm{a}}\right)$ absorbed by the silicon NW as shown in figure 2 (b), then the heat distribution will be longitudinally allowing us to use the formula: $\kappa=\frac{L}{2 . S} \cdot \frac{\Delta P_{a}}{\Delta T}$. We assumed that the increase in the laser power i.e. increasing the local temperature of the silicon NW does not affect the temperature of the heat sink and the local heat generated is transferred uniformly to the heat sink. With the help of linear fit of the $\omega \sim$ T graph i.e. $\omega=\omega_{0}+\alpha$.T , the above formula can be modified to $\kappa=\frac{4 . L}{2 . \pi d^{2}} \cdot \alpha \cdot\left(\frac{\delta \omega}{\delta P_{a}}\right)^{-1}$.

With laser spot diameter $0.5 \mu \mathrm{m}$, the diameter of silicon NW $112 \mathrm{~nm}$, length of silicon NW $\sim 3.4$ $\mu \mathrm{m}$, the value of first-order temperature coefficient $(\alpha)$ as $-0.01776 \mathrm{~cm}^{-1} \mathrm{~K}^{-1}$ and value of $\frac{\delta \omega}{\delta P_{a}}$ as $-0.05721 \mathrm{~cm}^{-1}(\mu \mathrm{W})^{-1}$, the thermal conductivity of the single silicon NW sample is estimated to be $\sim 53 \mathrm{~W} / \mathrm{m} . \mathrm{K}$.

Doerk et al. reported the variation of thermal conductivity with NW diameter and for the silicon $\mathrm{NW}$ with diameter $\sim 115 \mathrm{~nm}$, room temperature thermal conductivity is about $40 \mathrm{~W} / \mathrm{m} . \mathrm{K} .{ }^{23}$ Similar thermal conductivity values were also reported by Li et al. for individual NWs with a nearly similar diameter. ${ }^{14}$ Although our thermal conductivity estimation is in broad agreement with that of Doerk et al. ${ }^{23}$ and $\mathrm{Li}$ et al. ${ }^{14}$, our estimated value is slightly higher. Thermal conductivity of a material is highly dependent on the external strain which affects the phonon group velocity as well as specific heat. Equilibrium Molecular Dynamics calculations by Li et al. on thermal conductivity of silicon NWs as a function of strain reveal higher thermal conductivity under compressive strain and lower thermal conductivity under tensile strain as compared to the unstrained one. ${ }^{37-39}$ In addition, surface roughness causes more boundary scattering which greatly reduces the thermal conductivity of the silicon NW. ${ }^{40}$ Therefore, the small discrepancy in our thermal conductivity could be due to the existence of compressive strain on the silicon NW. The suppressed thermal conductivity of silicon NW as compared to the bulk silicon $(\kappa=148 \mathrm{~W} / \mathrm{m} \cdot \mathrm{K})^{41}$ is due to increased phonon boundary scattering.

CONCLUSIONS: In summary, Raman spectroscopy is employed to determine the thermal conductivity of the CVD grown free-standing silicon NW of diameter $112 \mathrm{~nm}$. Free-standing 
silicon NWs are obtained by transferring the NWs from the substrate to a carbon-coated copper TEM grid. Isolated individual NW can sometimes found to be suspended on ruptured carbon film where one end rests on the carbon film and another end lies on the copper grid. To minimize the role of contact on thermal conductivity we consider a suspended single NW of length much larger than the diameter of the laser spot. The temperature dependence of Raman spectra provides the first-order temperature coefficient $(\alpha)$ which is $-0.01776 \mathrm{~cm}^{-1} \mathrm{~K}^{-1}$ and laser-induced Raman spectra undergo a redshift which provides a slope of $-0.05721 \mathrm{~cm}^{-1}(\mu \mathrm{W})^{-1}$. By means of the first-order temperature coefficient $(\alpha)$ value and slope of the Raman peak position power graph, the thermal conductivity of the free-standing single silicon NW is found to be $\sim 53 \mathrm{~W} / \mathrm{m} . \mathrm{K}$.

\section{ACKNOWLEDGEMENTS:}

We acknowledge P. V. Satyam for extending the SEM facility and Puspendu Guha for assistance during the experiment.

\section{REFERENCES:}

1. Cui, Y. \& Lieber, C. M. Functional nanoscale electronic devices assembled using silicon nanowire building blocks. Science (80-. ). 291, 851-853 (2001).

2. Guerfi, Y. \& Larrieu, G. Vertical Silicon Nanowire Field Effect Transistors with Nanoscale Gate-All-Around. Nanoscale Res. Lett. 11, (2016).

3. Ghosh, R. \& Giri, P. K. Silicon nanowire heterostructures for advanced energy and environmental applications: A review. Nanotechnology 28, (2017).

4. Zhong, Z., Yang, C. \& Lieber, C. M. Silicon Nanowires and Nanowire Heterostructures. in Nanosilicon 176-216 (Elsevier Ltd, 2008). doi:10.1016/B978-008044528-1.50006-3.

5. Kim, J. et al. Graphene/Si-nanowire heterostructure molecular sensors. Sci. Rep. 4, (2014).

6. Hyun, J. K., Zhang, S. \& Lauhon, L. J. Nanowire Heterostructures. Annu. Rev. Mater. Res. 43, 451-479 (2013).

7. Oliva, M., Gao, G., Luna, E., Geelhaar, L. \& Lewis, R. B. Axial GaAs/Ga(As, Bi) nanowire heterostructures. Nanotechnology 30, (2019). 
8. Boukai, A. I. et al. Silicon nanowires as efficient thermoelectric materials. Nature 451, 168171 (2008).

9. Hicks, L. D. \& Dresselhaus, M. S. Effect of quantum-well structures on the thermoelectric figure of merit. Phys. Rev. B 47, 12727-12731 (1993).

10. Mahan, G., Sales, B. \& Sharp, J. Thermoelectric Materials: New Approaches to an Old Problem. Phys. Today 50, 42-47 (1997).

11. Chen, X., Wang, Y., Ma, Y., Cui, T. \& Zou, G. Origin of the High Thermoelectric Performance in Si Nanowires: A First-Principle Study. J. Phys. Chem. C 113, 14001-14005 (2009).

12. Ventura, G. \& Perfetti, M. How to Measure Thermal Conductivity. in 169-193 (2014). doi:10.1007/978-94-017-8969-1_8.

13. Ponomareva, I., Srivastava, D. \& Menon, M. Thermal conductivity in thin silicon nanowires: Phonon confinement effect. Nano Lett. 7, 1155-1159 (2007).

14. Li, D. et al. Thermal conductivity of individual silicon nanowires. Appl. Phys. Lett. 83, 2934-2936 (2003).

15. Cahill, D. G. Thermal conductivity measurement from 30 to $750 \mathrm{~K}$ : The $3 \omega$ method. Rev. Sci. Instrum. 61, 802-808 (1990).

16. Shamsa, M. et al. Thermal conductivity of diamond-like carbon films. Appl. Phys. Lett. 89, 20-23 (2006).

17. Choi, T.-Y., Poulikakos, D., Tharian, J. \& Sennhauser, U. Measurement of the Thermal Conductivity of Individual Carbon Nanotubes by the Four-Point Three- $\omega$ Method. Nano Lett. 6, 1589-1593 (2006).

18. Balandin, A. A. et al. Superior thermal conductivity of single-layer graphene. Nano Lett. 8, 902-907 (2008).

19. Sahoo, S., Gaur, A. P. S., Ahmadi, M., Guinel, M. J. F. \& Katiyar, R. S. Temperaturedependent Raman studies and thermal conductivity of few-layer MoS2. J. Phys. Chem. C 
117, 9042-9047 (2013).

20. Ghosh, S. et al. Extremely high thermal conductivity of graphene: Prospects for thermal management applications in nanoelectronic circuits. Appl. Phys. Lett. 92, 1-4 (2008).

21. Chen, S. et al. Thermal conductivity of isotopically modified graphene. Nat. Mater. 11, 203-207 (2012).

22. Sahoo, S., Chitturi, V. R., Agarwal, R., Jiang, J. W. \& Katiyar, R. S. Thermal conductivity of freestanding single wall carbon nanotube sheet by Raman spectroscopy. ACS Appl. Mater. Interfaces 6, 19958-19965 (2014).

23. Doerk, G. S., Carraro, C. \& Maboudian, R. Single nanowire thermal conductivity measurements by Raman thermography. ACS Nano 4, 4908-4914 (2010).

24. Cahill, D. G. Analysis of heat flow in layered structures for time-domain thermoreflectance. Rev. Sci. Instrum. 75, 5119-5122 (2004).

25. Li, Q., Liu, C., Wang, X. \& Fan, S. Measuring the thermal conductivity of individual carbon nanotubes by the Raman shift method. Nanotechnology 20, (2009).

26. Beechem, T., Yates, L. \& Graham, S. Invited review article: Error and uncertainty in Raman thermal conductivity measurements. Rev. Sci. Instrum. 86, (2015).

27. Lysenko, V., Perichon, S., Remaki, B., Barbier, D. \& Champagnon, B. Thermal conductivity of thick meso-porous silicon layers by micro-Raman scattering. J. Appl. Phys. 86, 6841-6846 (1999).

28. Cao, F. \& He, Z. Determination of thermal conductivity using micro-Raman spectroscopy with a three-dimensional heating model. J. Raman Spectrosc. 50, 1969-1976 (2019).

29. Malekpour, H. \& Balandin, A. A. Raman-based technique for measuring thermal conductivity of graphene and related materials. J. Raman Spectrosc. 49, 106-120 (2018).

30. Doerk, G. S., Carraro, C. \& Maboudian, R. Temperature dependence of Raman spectra for individual silicon nanowires. Phys. Rev. B - Condens. Matter Mater. Phys. 80, 1-4 (2009). 
31. Hart, T. R., Aggarwal, R. L. \& Lax, B. Temperature dependence of Raman scattering in silicon. Phys. Rev. B 1, 638-642 (1970).

32. Klemens, P. G. Anharmonic decay of optical phonons. Phys. Rev. 148, 845-848 (1966).

33. Su, Z. et al. Temperature-Dependent Raman Scattering of Silicon Nanowires. J. Phys. Chem. B 110, 1229-1234 (2006).

34. Palik, E. Handbook of optical constants of solids. (1998).

35. Aspnes, D. E. \& Studna, A. A. Dielectric functions and optical parameters of Si, Ge, GaP, GaAs, GaSb, InP, InAs, and InSb from 1.5 to $6.0 \mathrm{eV}$. Phys. Rev. B 27, 985-1009 (1983).

36. Piscanec, S. et al. Raman spectroscopy of silicon nanowires. Phys. Rev. B 68, 241312 (2003).

37. Li, X., Maute, K., Dunn, M. L. \& Yang, R. Strain effects on the thermal conductivity of nanostructures. Phys. Rev. B - Condens. Matter Mater. Phys. 81, 1-11 (2010).

38. Murphy, K. F., Piccione, B., Zanjani, M. B., Lukes, J. R. \& Gianola, D. S. Strain- and Defect-Mediated Thermal Conductivity in Silicon Nanowires. Nano Lett. 14, 3785-3792 (2014).

39. Gan, M., Samvedi, V. \& Tomar, V. Raman spectroscopy-based investigation of thermal conductivity of stressed silicon microcantilevers. J. Thermophys. Heat Transf. 29, 845-857 (2015).

40. Bandaru, P. R. \& Pichanusakorn, P. An outline of the synthesis and properties of silicon nanowires. Semiconductor Science and Technology vol. 25 (2010).

41. Maycock, P. D. Thermal conductivity of silicon, germanium, III-V compounds and III-V alloys. Solid State Electron. 10, 161-168 (1967). 